\title{
Parlamentarische Untersuchungsausschüsse im Deutschen Bundestag - bloßes Skandalisierungsinstrument der Opposition?
}

\author{
Matthias Riede und Henrik Scheller
}

Parlamentarische Untersuchungsausschüsse sollen durch Aufklärung von institutionellem und personellem Fehlverhalten im öffentlichen Leben einen Beitrag zur Selbstinformation des Parlaments leisten. ${ }^{1}$ In der politischen Debatte steht allerdings immer wieder der Vorwurf im Raum, dass sie der Opposition lediglich zur „Gängelung“ und „Skandalisierung“ der jeweils amtierenden Regierung und der sie tragenden Mehrheitsfraktionen dienen.

Da Skandale als Instrumente und Mechanismen sozialer Kontrolle per se eine breite Öffentlichkeit erfordern, ist das Wechselspiel zwischen politischen Akteuren und Medien von zentraler Bedeutung. Im Mittelpunkt der folgenden Analyse steht deshalb die begleitende Berichterstattung in ausgewählten Printmedien über die Arbeit der Parlamentarischen Untersuchungsausschüsse des Deutschen Bundestages im Zeitraum von 1990 bis 2011. Dabei wird von der These ausgegangen, dass zwar die Mehrzahl der Untersuchungsausschüsse tatsächlich als Instrument der jeweiligen Opposition genutzt wurde, um unter dem Kalkül der erhöhten Medienberichterstattung die regierenden Mehrheitsfraktionen zu skandalisieren. Allerdings gab es auch Fälle, in denen sich diese Logik in ihr Gegenteil verkehrte, da Untersuchungsausschüsse auch von den Skandalisierten als Forum medialer Selbstinszenierung genutzt wurden. Voraussetzung einer erfolgreichen Skandalisierung über die Medien - so die zweite hier vertretene These - ist jedoch immer die öffentliche Tagung von Untersuchungsausschüssen und die Prominenz der geladenen Zeugen.

Zur vergleichenden Analyse spezifischer Skandalverläufe und -logiken wird hier auf das theoretische Konzept der medialen Thematisierung in Zyklen zurückgegriffen. ${ }^{2}$ Weitere theoretische Überlegungen zu den grundsätzlichen Rahmenbedingungen von Skandalen liefern Esser und Hartung. ${ }^{3}$ Die empirische Analyse zur medialen Berichterstattung über die Parlamentarischen Untersuchungsausschüsse beruht dabei auf einer quantitativen Erhebung ausgewählter überregionaler Printmedien. Indem phasenweise das Aufkommen von Zeitungsberichten, Kommentaren und Leitartikeln in Bezug zu den Ereignissen im jeweiligen Ausschuss gesetzt wird, wird die Analyse punktuell um qualitative Aspekte ergänzt. Dabei richtet sich das Augenmerk nicht nur auf die idealtypischen Beispiele, wie die Untersuchungsausschüsse „Kommerzielle Koordinierung“, „Treuhandanstalt“, „Rechtsextremistische Vorkommnisse an Standorten der Bundeswehr in Hamburg“, „Wahlbetrug“, „Sicherheitsrisiko Visapolitik“. Vielmehr sollen vor allem solche Fälle analysiert werden, die atypische Skandalisierungsverläufe („Plutonium-Schmuggel“, „DDR-Vermögen“, „Hypo-Real-Esta-

1 Vgl. Hans-Peter Schneider, Spielregeln für den investigativen Parlamentarismus. Das neue Untersuchungsausschuss-Gesetz des Bundestages, in: Neue Juristische Wochenschrift, 54. Jg. (2001), H. 36, S. $2604-2608$.

2 Vgl. Steffen Kolb, Mediale Thematisierung in Zyklen: Theoretischer Entwurf und empirische Anwendung, Köln 2005.

3 Vgl. Frank Esser / Uwe Hartung, Skandale als Spiegel der politischen Kultur in Deutschland. Konstruktionen und Berichterstattungsmuster, in: Klaus Kamps / Jörg-Uwe Nieland (Hrsg.), Regieren und Kommunikation, Meinungsbildung, Entscheidungsfindung und gouvernementales Kommunikationsmanagement - Trends, Vergleiche, Perspektiven, Köln 2006, S. 305 - 336. 
te") oder keine beziehungsweise nur wenige Skandalmerkmale aufweisen („HIV-Infektionen durch Blut und Blutprodukte“ und „Gorleben“). Dabei sind die Gründe für den abweichenden Verlauf von besonderem Interesse, um Aufschluss darüber zu erlangen, unter welchen Bedingungen Medienberichterstattung und Ausschussarbeit miteinander korrespondieren.

\section{Politische Skandale in der Mediengesellschaft: ein Phasenmodell}

Politische Skandale erfüllen eine Funktion sozialer Kontrolle. ${ }^{4}$ Ihr zentrales Merkmal bildet die „kollektive Empörung“ über eine Verletzung gesellschaftlich anerkannter Normen und Werte durch Vertreter der Eliten, die meist nicht unmittelbar durch Gesetz sanktioniert werden können. ${ }^{5}$ Sie dienen hauptsächlich der „Ausübung von politischer Herrschaftskontrolle“6 und der „Auseinandersetzung um Macht, Geld und Reputation“ - sei es durch politische Akteure, gesellschaftliche Institutionen oder die Medien. ${ }^{7}$ Skandale erfüllen damit auch eine Konflikt- und Kampffunktion, denn nicht selten dient die Skandalisierung einzelner politischer Akteure oder ganzer Regierungen der Opposition zur Veränderung bestehender Machtverhältnisse.

In ihrer Analyse zu Ursachen und Verläufen politischer Skandale nennen Esser und Hartung drei Umstände, die gesellschaftliche Skandale auslösen können: Erstens, wenn in einem Konflikt zwischen zwei Wertegemeinschaften „ein Defizit oder Fehlverhalten (...) instrumentalisiert werden kann“; zweitens, wenn die folgende Skandalisierung in „der Auseinandersetzung um Macht“ genutzt werden kann oder drittens, wenn es Menschen möglich wird, „politische Herrschaft bloßzustellen und ihre, wahren' Motive offen zu legen". ${ }^{8}$ Um Skandalisierungsverläufe und -muster zu untersuchen, kann auf das Zyklen-Modell medialer Thematisierung von Kolb zurückgegriffen werden, das sich für die Analyse öffentlicher und politischer Skandale gleichermaßen eignet. ${ }^{9}$ Kolb unterscheidet zwischen fünf Phasen eines idealtypischen Verlaufs öffentlicher Skandale: der Latenz-, der Aufschwung-, der Etablierungs-, der Abschwung- sowie der Marginalisierungs- beziehungsweise Rehabilitationsphase. ${ }^{10}$

In der Latenzphase wird vereinzelt in einigen Medien über das den Skandal bestimmende Thema berichtet. Insgesamt findet im Vergleich zu den folgenden Phasen aber eine spärliche Berichterstattung statt, da das „Experteninteresse und (der) wissenschaftliche Austausch"11 im Vordergrund stehen. Zudem entwickelt das Thema in dieser Phase noch keine Eigendynamik. Der Durchbruch zu einem öffentlichen Skandal ist nicht garantiert. Dies ändert sich in der Regel mit dem Eintreten eines so genannten Schlüsselereignisses, das zugleich das Ende der Latenzphase markiert. Hierdurch wird der Weg aus den „Fachpubli-

4 Vgl. ebenda, S. 310.

5 Vgl. Steffen Burkhard, Medienskandale. Zur moralischen Sprengkraft öffentlicher Diskurse, Köln 2006, S. 36.

6 Burghard Oeverhaus, Der parlamentarische Untersuchungsausschuss. Eine Untersuchung seiner Funktion am Beispiel von politisch-ökonomischen Skandalen in Berlin, Berlin / München 2006, S. 37.

7 Vgl. Frank Esser / Uwe Hartung, a.a.O. (Fn. 3), S. 311.

8 Ebenda, S. 330.

9 Vgl. Steffen Kolb, a.a.O. (Fn. 2); ähnlich aber auch Steffen Burkhard, a.a.O. (Fn. 5).

10 Vgl. Steffen Kolb, a.a.O. (Fn. 2), S. 97.

11 Ebenda, S. 80. 
kationen heraus in die Publikumsmedien"12 geebnet. Schlüsselereignisse sind hinreichende, jedoch nicht notwendige Bedingungen für die Entstehung eines Skandals.

In der Aufschwungphase erlebt das Thema durch das Übergreifen auf immer weitere Medien eine immer stärkere öffentliche Aufmerksamkeit. Einzelne Akteure versuchen dabei, die Definitions- und Deutungshoheit zu erlangen, indem sie ein (vermeintliches) Problem zu einem öffentlich beziehungsweise politisch relevanten Thema hochstilisieren, um so nicht zuletzt sich selber in der medialen Auseinandersetzung zu positionieren. Die Aufschwungphase endet, wenn die mediale Berichterstattung ihren Höhepunkt erreicht. Der anschließende Übergang in die Etablierungsphase geht meist nicht mit einem spezifischen Ereignis einher. Die überregionalen Medien haben das Thema vielmehr in ihre Berichterstattung fest integriert, und es lässt sich „die größte Publikationsdichte pro Zeiteinheit"13 konstatieren. Eine weitere Zunahme erklärt sich dann vor allem durch das Übergreifen auf regionale und lokale Medien. In der Öffentlichkeit kommt es durch die Verfestigung eines zunehmend dominierenden Meinungsbildes zu einer Art Entscheidungsfindung, in der „individuelles Abweichen vom sozialen Verhaltens-, Moral- und Wertekodex verhandelt wird" ${ }^{14}$.

Der Höhepunkt der Skandalisierungskurve wird in der Regel dann erreicht, wenn es zu einer politischen Entscheidung beziehungsweise zur „Lösung des Problems“ kommt. ${ }^{15}$ Dies erfolgt häufig in Form einer öffentlichen Entschuldigung des Skandalisierten. Die Abschwungphase, die durch einen Rückgang der medialen Berichterstattung und eine damit einhergehende Abnahme des allgemeinen öffentlichen Interesses gekennzeichnet ist, kann erst nach diesem Ereignis eintreten. Manchmal erfolgt dies auch nur durch eine „vorgeschobene Lösung des Problems" "16. In der Abschwungphase kommt es zu einer Qualifizierung der Problemlösung sowie einer Retrospektive des Skandals durch die Medien. Die Rolle des Skandalisierten spielt hierbei eine wichtige Rolle, denn sein Verhalten muss von der Öffentlichkeit und den Medien als angemessen eingestuft werden. Nur dann kann es zur Rehabilitation kommen. Allerdings können in dieser Phase auch ,angrenzende Themen “ und Personen in den Mittelpunkt des medialen Interesses rücken. ${ }^{17}$ Der zweite Wendepunkt auf der Skandalisierungskurve wird durch den Übergang von der Abschwung- in die Marginalisierungs- beziehungsweise Rehabilitationsphase markiert. Die mediale Berichterstattung nimmt in dieser Phase am stärksten ab, und neue Themen gewinnen an Bedeutung. Der Skandal wird auf diese Weise „marginalisiert“ und die gesellschaftliche Reputation des Skandalisierten sukzessive wieder hergestellt.

Grundvoraussetzung für diese Skandalisierungslogik bildet der enge „Interaktions- und Handlungszusammenhang “ zwischen Politik und Medien, der verschiedentlich als wechselseitiges Abhängigkeitsverhältnis beschrieben wird. ${ }^{18}$ Während Politiker zur Legitimierung

12 Ebenda, S. 81.

13 Steffen Burkhard, a.a.O. (Fn. 5), S. 197.

14 Ebenda, S. 199.

15 Vgl. Steffen Kolb, a.a.O. (Fn. 2), S. 83.

16 Ebenda, S. 83.

17 Vgl. ebenda, S. 83.

18 Vgl. Ulrich Sarcinelli, Politische Kommunikation in Deutschland. Zur Politikvermittlung im demokratischen System, Wiesbaden 2005, S. 111. Meyer sieht dieses gegenseitige Abhängigkeitsverhältnis nicht gegeben und spricht stattdessen von einer „Kolonisierung“ des politischen Systems durch die Medien. Siehe Thomas Meyer, Mediokratie. Auf dem Weg in eine andere Demokratie?, in: APuZ, B 15-16/2002, S. 7. 
ihres eigenen Handelns auf Publizität angewiesen sind, benötigen Medien - mit Blick auf den hart umkämpften Nachrichtenmarkt - möglichst frühzeitig und exklusiv Informationen. Aufgrund der zentralen Bedeutung der Kommunikation für die politischen Akteure unterscheidet Sarcinelli zwischen „Darstellungs-“ und „Entscheidungspolitik“. Darstellungspolitik zeichne sich dabei durch ein Interesse an „politischen Momentaufnahmen und dem Augenblickserfolg "19 aus. Für die politischen Akteure bilden die Massenmedien deshalb ein „notwendiges Herrschaftsmittel“ und eine „Machtprämie“ ${ }^{20}$ Damit gehe auch eine professionelle "Selbstmediatisierung der Politik“ einher, die sowohl in qualitativer als auch in quantitativer Hinsicht zu einer der „Hauptaktivitäten des politischen Systems geworden“21 sei. Die Kommunikation politischer Akteure diene mithin der Darstellung eigener Anliegen und Erfolge, indem die herrschende Medienlogik gezielt antizipiert werde. Vor diesem Hintergrund und aufgrund der Kurzlebigkeit einzelner Themen in der Berichterstattung spielen Formen der Inszenierung, Dramatisierung und Personalisierung - vor allem in der Auseinandersetzung mit dem politischen Gegner - eine entscheidende Rolle. Verschiedentlich wird die politische Auseinandersetzung deshalb als symbolisches „Politainment“ beschrieben und kritisiert - einer Form der „massenmedialen Kommunikation“, in der politische Themen, Akteure, Prozesse und Deutungsmuster „im Modus der Unterhaltung, zu einer neuen Realität des Politischen montiert werden“22. Damit gehe eine personalisierte, „mediendramaturgische" Berichterstattung einher, die sich durch die Konzentration auf einige wichtige Ereignisse und Spitzenakteure auszeichne. ${ }^{23}$

\section{Parlamentarische Untersuchungsausschüsse: rechtliche Grundlagen}

Die herausgehobene Stellung von Untersuchungsausschüssen im parlamentarischen System der Bundesrepublik ist in Art. 44 GG normiert. Näheres regelt das seit 2001 geltende „Gesetz zur Regelung des Rechts der parlamentarischen Untersuchungsausschüsse im Deutschen Bundestag“ (PUAG) in Verbindung mit der Geschäftsordnung des Bundestages. ${ }^{24}$ Danach sind Parlamentarische Untersuchungsausschüsse nicht-ständige Ausschüsse, die auf Verlangen eines Viertels der Abgeordneten eingesetzt werden müssen und mit umfangreichen Untersuchungs- und Kontrollbefugnissen ausgestattet sind. ${ }^{25}$ Sie haben das Recht, sich mit allen Vorgängen zu befassen, die in den Kompetenzbereich von Parlament und Regierung fallen. ${ }^{26}$ Eine politische Bewertung des untersuchten Gegenstandes steht Untersuchungsausschüssen zwar selber nicht explizit zu - die so genannten Schlussfolgerungen in entspre-

19 Ulrich Sarcinelli, a.a.O. (Fn. 18), S. 116.

20 Ebenda, S. 117.

21 Thomas Meyer, a.a.O. (Fn. 18), S. 12.

22 Ebenda, S. 8; Andreas Dörner, Politainment. Politik in der medialen Erlebnisgesellschaft, Berlin 2001, S. 31.

23 Vgl. Ulrich Sarcinelli, a.a.O. (Fn. 18), S. 121.

$24 \$ 54$, Abs. 2 GO-BT: „Soweit das Grundgesetz oder Bundesgesetze die Einsetzung von Ausschüssen vorschreiben oder zulassen, richtet sich die Einsetzung und das Verfahren nach den Bestimmungen dieser Geschäftsordnung, es sei denn, dass im Grundgesetz, in den Bundesgesetzen oder in besonderen Geschäftsordnungen etwas anderes bestimmt ist."

25 Vgl. Dieter Wiefelspütz, Das Untersuchungsausschussgesetz, Baden-Baden 2003, S. 28.

26 Vgl. Burghard Oeverhaus, a.a.O. (Fn. 6), S. 30. 
chenden Abschlussberichten erfüllen jedoch faktisch diese Funktion. ${ }^{27}$ Untersuchungsausschüsse können zur Selbstinformation des Parlaments eigenständig „,inquisitorisch“ tätig werden, um nicht auf Regierungsinformationen angewiesen zu sein. In Verbindung mit den gesetzlich garantierten Sonderrechten der Opposition in der Verfahrensordnung und der Beweisaufnahme macht sie dies zum schärfsten parlamentarischen Kontrollinstrument gegenüber Regierung und Mehrheitsfraktionen. ${ }^{28}$

Parlamentarische Untersuchungsausschüsse tagen grundsätzlich öffentlich. Auf Antrag kann die Öffentlichkeit jedoch ausgeschlossen werden. Sie sind ausführendes Organ und nehmen die Rechte des Parlaments wahr. Qua Verfassung ist formal das Bundestagsplenum Träger des Untersuchungsrechts und Herr des Untersuchungsverfahrens. ${ }^{29}$ Planung und Durchführung der Untersuchung selbst obliegen jedoch dem vom Plenum legitimierten Ausschuss. ${ }^{30}$ Grundsätzlich kann zwischen vier Untersuchungstypen unterschieden werden: Kontroll-, Gesetzgebungs-, Kollegial- und Skandal- beziehungsweise Missstandsenqueten. ${ }^{31}$ Am häufigsten hat der Bundestag bisher Kontrollenqueten eingesetzt. Hierbei steht die Kontrolle von Regierung und Verwaltung im Mittelpunkt. Während Gesetzgebungsenqueten Vorlagen und Empfehlungen zu bestimmten Reformvorhaben erarbeiten sollen, beschäftigen sich Kollegialenqueten mit innerparlamentarischen Ordnungsfragen und dem Verhalten einzelner Abgeordneter. Skandal- und Missstandsenqueten widmen sich hingegen den Ursachen von Missständen im staatlichen und nichtstaatlichen Bereich.

\section{Untersuchungsausschüsse seit der deutschen Wiedervereinigung}

Zu dreizehn der vierzehn seit 1990 vom Bundestag eingesetzten Untersuchungsausschüssen wurde die mediale Berichterstattung analysiert (mit dem Fall Murat Kurnaz beschäftigten sich zwei Untersuchungsausschüsse - hier wird nur der BND-Untersuchungsausschuss beleuchtet, da der parallel tagende Untersuchungsausschuss des Verteidigungsausschusses weitgehend geheim tagte) ${ }^{32}$ : „Kommerzielle Koordinierung“ (1991 bis 1994), „Treuhandanstalt“ (1993 bis 1994), „HIV-Infektionen durch Blut und Blutprodukte“ (1993 bis 1994), „Plutonium-Schmuggel“ (1995 bis 1998), „DDR-Vermögen“ (1995 bis 1998), „Rechtsextremistische Vorkommnisse an der Führungsakademie der Bundeswehr in Hamburg“ (1998), „Parteispenden“ (1999 bis 2002), „Wahlbetrug“ (2002 bis 2003), „Sicherheitsrisiko Visapolitik“ (2004 bis 2005), „BND“ zusammen mit dem Fall Murat Kurnaz (2006 bis 2009), „Hypo-Real-Estate“ (2009), „Kundus“ (2009 bis 2011) und „Gorleben“ (seit 2010). Als Hauptindikator für einen möglichen Skandal gilt dabei die Anzahl der in überregionalen

27 Vgl. Wolfgang Ismayr, Der Deutsche Bundestag, Wiesbaden 2012, S. 362.

28 Vgl. Lars Brocker / Paul Glauben, Das Recht der parlamentarischen Untersuchungsausschüsse in Bund und Ländern. Ein Handbuch, Köln 2011, S. 3.

29 Vgl. ebenda, S. 10. Eine „exklusive Zuständigkeit“ kommt nach Art. 45 Abs. 2 GG dem Verteidigungsausschuss zu. Dieser kann sich auf Antrag eines Viertels seiner Mitglieder selbst zum Untersuchungsausschuss konstituieren. Vgl. dazu Dieter Engels, Parlamentarische Untersuchungsausschüsse. Grundlagen und Praxis im Deutschen Bundestag, Heidelberg 1991, S. 33.

30 Vgl. Lars Brocker / Paul Glauben, a.a.O. (Fn. 28), S. 2.

31 Vgl. Dieter Engels, a.a.O. (Fn. 29), S. 12 - 14.

32 Der Untersuchungsausschuss „Terrorgruppe nationalsozialistischer Untergrund“, der seine Tätigkeit 2012 aufnahm, konnte hier nicht berücksichtigt werden. 
Printmedien erschienenen Beiträge über den jeweiligen untersuchten Ausschuss pro Zeiteinheit. Dabei wurden folgende Medien berücksichtigt: Süddeutsche Zeitung, Das Parlament, Handelsblatt, Frankfurter Allgemeine Zeitung, Frankfurter Allgemeine Sonntagszeitung, Frankfurter Rundschau, Bildzeitung, Bild am Sonntag, Die Welt, Welt am Sonntag, taz.die tageszeitung, Financial Times Deutschland, Die Zeit, Focus, Der Spiegel, Stern, Der Freitag. Zur Erhebung der Presseberichterstattung wurde eine Sequenzierung in Monatsabschnitte vorgenommen. ${ }^{33}$ Mit Blick auf die Erklärung der einzelnen Ausschläge, die vor allem einen atypischen Skandalverlauf indizieren, wird die Bedeutung der Personalisierung in den Medien problematisiert und der Frage nachgegangen, inwieweit skandalisierte Akteure Untersuchungsausschüsse möglicherweise selber zur Eigenverteidigung, Selbstprofilierung sowie zum Angriff auf die Opposition nutzten.

\subsection{Idealtypische Skandalverläufe}

Die hier analysierten Ausschüsse erreichten - abgesehen von drei Ausnahmen - ihren Höhepunkt an öffentlicher Aufmerksamkeit jeweils gleich zu Beginn des Untersuchungszeitraums. ${ }^{34}$ Fünf der Ausschüsse zeigten dabei nach Erreichen des Maximalwertes keine besonderen Ausschläge mehr und können somit als typische Skandalverläufe im Sinne der theoretischen Annahmen von Kolb klassifiziert werden. ${ }^{35}$

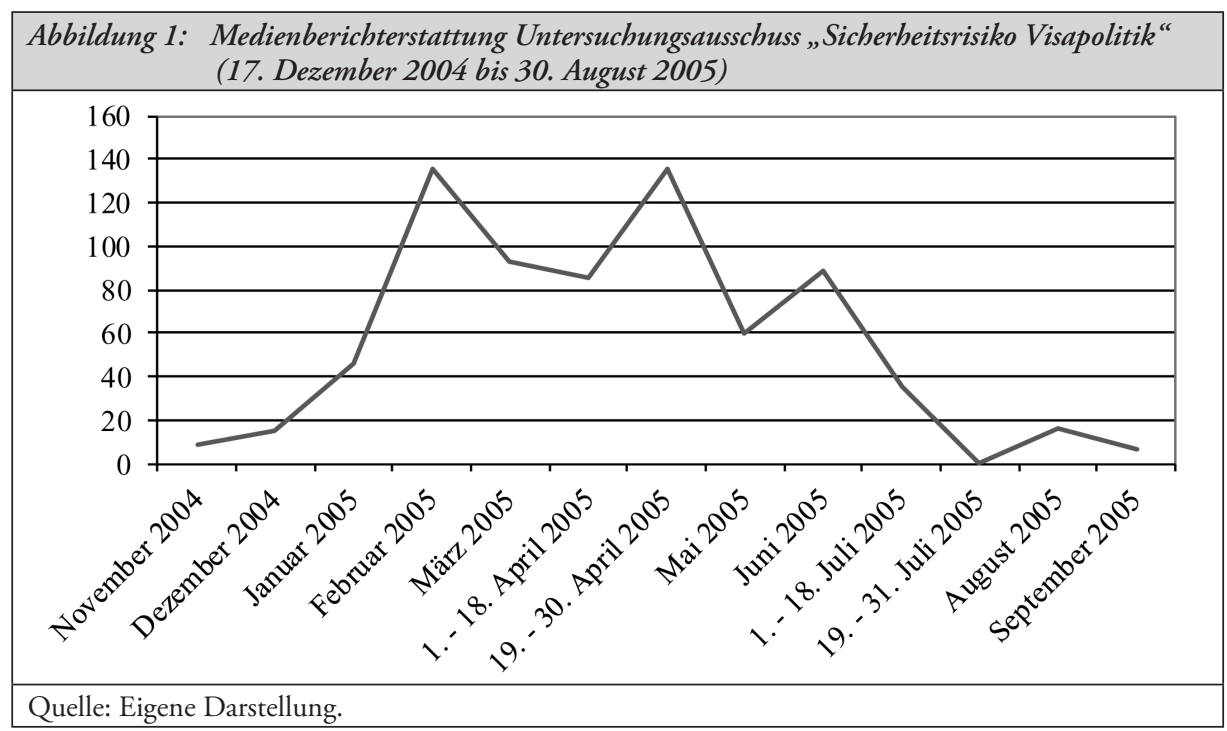

33 Bei besonderen Ereignissen, wie etwa Zeugenaussagen prominenter Akteure, wurden auch monatsübergreifende Zeiträume gewählt.

34 Die drei Ausnahmen sind die Untersuchungsausschüsse „Hypo-Real-Estate“ (vgl. Abbildung 6), „Plutonium-Schmuggel“ (vgl. Abbildung 7) und „DDR-Vermögen“ (vgl. Abbildung 8).

35 Im Einzelnen handelt es sich dabei um die Untersuchungsausschüsse „Kommerzielle Koordinierung“ (vgl. Abbildung 2), „Rechtsextremistische Vorkommnisse an Standorten der Bundeswehr in Hamburg“ (vgl. Abbildung 3), „Treuhandanstalt“ (vgl. Abbildung 4), „Wahlbetrug“ (vgl. Abbildung 5) und „Sicherheitsrisiko Visapolitik“ (vgl. Abbildung 1). 

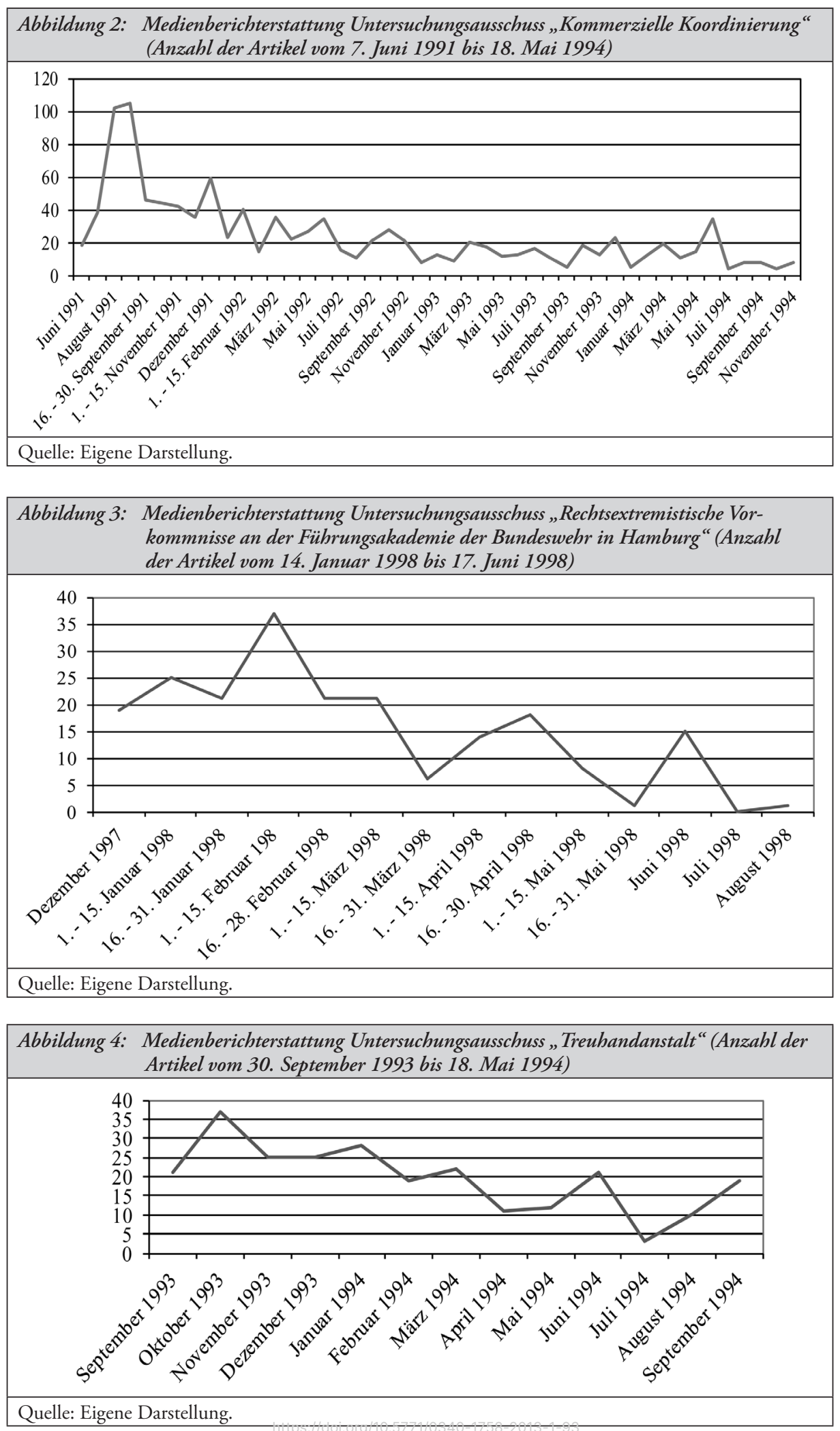


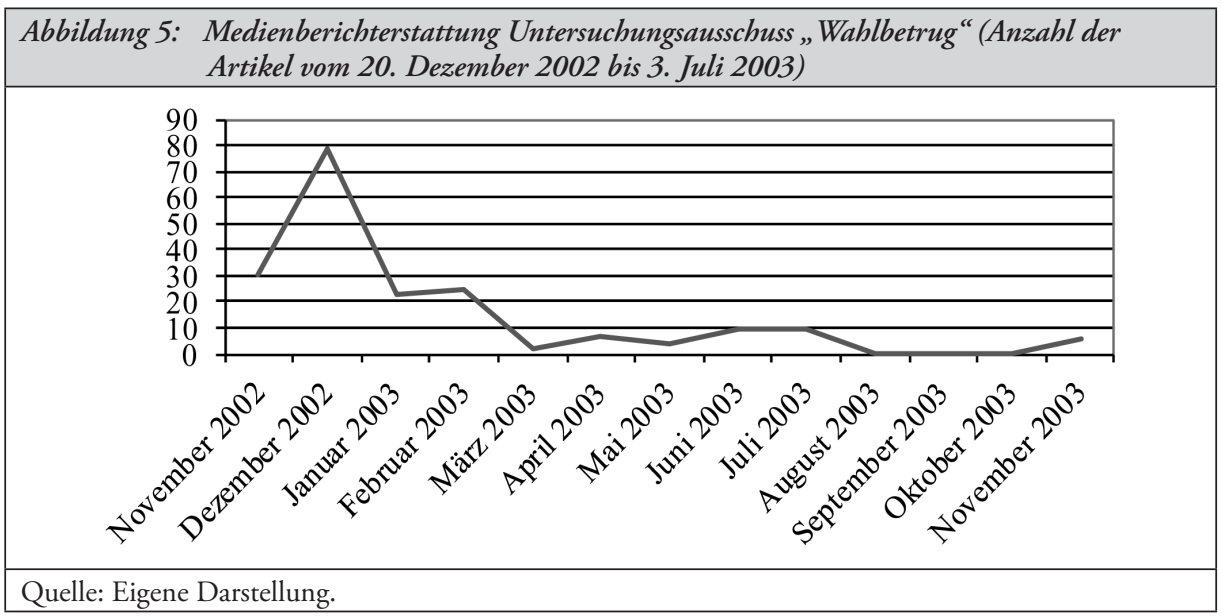

Der Untersuchungsausschuss „Sicherheitsrisiko Visapolitik“ - als Beispiel für einen idealtypischen Skandalverlauf - war Teil der so genannten Visa-Affäre um die Visa-Vergabepraxis der rot-grünen Bundesregierung unter Bundeskanzler Gerhard Schröder (SPD). Im Jahr 2000 erließ der damalige Staatsminister im Auswärtigen Amt, Ludger Volmer (Bündnis 90/Die Grünen), einen Runderlass zur Regelung des „Verfahrens zur Erteilung für die Einreise nach Deutschland“. Damit wurde der Ermessensspielraum bei der Visa-Vergabe der Konsularabteilungen deutscher Botschaften im Ausland ausgeweitet. Anfang 2004 wurden jedoch Missbrauchsfälle der neuen Vergabepraxis bekannt, in die offenbar Schleuserbanden in Osteuropa verwickelt waren. So war es in der deutschen Botschaft in Kiew zuvor zu einem auffälligen Anstieg an Visavergaben gekommen.

Die Latenzphase dieses Skandals setzte Anfang November 2004 ein, nachdem sich in den beiden vorangegangenen Monaten entsprechende Medienberichte gehäuft hatten. Die CDU/CSU, die damals die stärkste Oppositionsfraktion im Bundestag stellte, erwog in dieser Zeit auch die Einsetzung eines Untersuchungsausschusses zur fehlerhaften Umsetzung der LKW-Maut, entschied sich jedoch im November für die Untersuchung des Visamissbrauchs. ${ }^{36}$ Nach Bekanntgabe dieses Vorhabens durch die Fraktionsspitze erschienen im November 2004 insgesamt acht Berichte in den überregionalen Printmedien. Der Vorgang wurde dadurch zwar einem breiten Publikum bekannt, jedoch in den Medien noch nicht kontrovers diskutiert. Dieses änderte sich mit Eintreten des Schlüsselereignisses. Wie auch hier, fällt dies in den meisten Fällen mit der formalen Einsetzung eines Untersuchungsausschusses zusammen. So wurde der Visa-Untersuchungsausschuss am 18. Dezember 2004 eingesetzt. Der entsprechende Antrag der CDU/CSU-Bundestagsfraktion sah eine Klärung der Frage vor, ob durch Mitglieder der Bundesregierung oder in ihrem Verantwortungsbereich „durch die Visaverteilungspraxis (...) gegen geltendes Recht oder Internationale Verpflichtungen der Bundesrepublik Deutschland verstoßen wurde, (...) Schwarzarbeit, Prostitution, Frauenhandel, terroristische Handlungen oder sonstige Kriminalität (...) ermöglicht

36 Vgl. Johannes Leithäuser / Peter Carstens, Unionsfraktion beantragt Untersuchungsausschuss zur Visapraxis, in: FAZ vom 10. November 2004, S. 2. 
oder erleichtert wurden oder (...) auf sonstige Weise die Sicherheit der Bundesrepublik Deutschland oder anderer Schengenstaaten (...) gefährdet wurde“ 37 .

Während dieses Schlüsselereignis selber nur fünfzehn Mal in überregionalen Zeitungsbeiträgen dokumentiert wurde, nahm die Berichterstattung in der Folge deutlich zu. Im Januar 2005 erschienen bereits 46 Artikel. In dieser Aufschwungphase zeigt sich ein Phänomen, das bei nahezu allen hier untersuchten Ausschüssen in vergleichbar Weise zu Tage trat: eine über die Medien ausgetragene Debatte über mögliche Zeugen, die im Ausschuss aussagen sollen. Die Diskussion zwischen den Fraktionen konzentriert sich dabei stets auf prominente Politiker und hochrangige Beamte. Im Visa-Untersuchungsausschuss handelte es sich um den damaligen Außenminister Joschka Fischer und seinen Parlamentarischen Staatssekretär Ludger Volmer (beide Bündnis 90/Die Grünen), die beide für das späte Eingreifen des Auswärtigen Amtes zur Behebung der Missstände bei der Visa-Vergabepraxis verantwortlich gemacht wurden.

Das Ziel einer Skandalisierung Fischers, der sich zu dieser Zeit in repräsentativen Bevölkerungsumfragen großer Popularität erfreute, wurde dabei von Oppositionspolitikern offen genannt. So äußerte der Obmann der CDU/CSU-Fraktion im Untersuchungsausschuss, Jürgen Gehb, gegenüber der tageszeitung, dass man „es nicht mit Trauer hinnehmen“ würde, sollte „Herr Fischer durch den Ausschuss beschädigt“ 38 werden. Allerdings befürwortete auch Fischers eigene Fraktion eine Aussage, um „bei der Aufklärung der Vorwürfe (zu) helfen und zu einer Versachlichung der Debatte “ 39 beizutragen. Dies zeigt, dass auch die Mehrheitsfraktionen versuchen, Untersuchungsausschüsse mit Hilfe einer Personalisierung proaktiv zur Selbstverteidigung zu nutzen. Mit dieser Strategie blieb Fischer jedoch - neben Helmut Kohl während des CDU-Parteispendenskandals und Frank-Walter Steinmeier (SPD) im „Fall Murat Kurnaz" - eine der wenigen Ausnahmen.

Im Februar 2005 nahm die Berichterstattung über die Ausschussarbeit weiter zu. Dabei entwickelte sich die Auseinandersetzung über den Zeitpunkt von Fischers Aussage zu einer machtpolitischen Kraftprobe zwischen Mehrheitsfraktionen und Opposition. Mit Blick auf die nordrhein-westfälische Landtagswahl am 22. Mai 2005 wollte die Opposition den Außenminister möglichst rasch verhören. Die Regierungsfraktionen wollten diesen Termin jedoch möglichst lang aufschieben, da Fischers Ansehen in der Öffentlichkeit zu sinken begann und sich die Umfragewerte von Bündnis 90/Die Grünen verschlechterten. ${ }^{40}$ Die Berichterstattung über den Ausschuss wies Ende Februar/Anfang März 2005 ein konstant hohes Niveau auf. Der Skandal war nun in der Etablierungsphase angelangt und steuerte auf seinen Höhepunkt zu - den Aussagen Fischers und Volmers, die live im Fernsehen übertragen werden sollten. ${ }^{41}$ Dieses Vorhaben wurde im Vorfeld intensiv von den Printmedien diskutiert, da es sich dabei um ein Novum in der Bundestagsgeschichte handelte. Seinen eigentlichen Höhepunkt erreichte der Skandal zwischen dem 18. und 30. April 2005. In diesem Zeitraum erschienen in den untersuchten Printmedien 135 Artikel, die sich mit dem Untersuchungsausschuss beschäftigten. Volmer übernahm am 21. April 2005 vor dem Ausschuss eine Mitverantwortung für den Runderlass: „Um es noch einmal zusammenzufassen:

37 BT-Drucksache 15/4285, S. 2.

38 Ulrike Herrmann, Union will mit Visa Joschka Fischer besiegen, in: taz vom 21. Januar 2005, S. 7.

39 Daniel Riegger, Versuch der Skandalisierung, in: FR vom 26. Februar 2005, S. 9.

40 Vgl. Carsten Fiedler, „Causa Fischer“ belastet die Grünen, in: Die Welt vom 24. Februar 2005.

41 Vgl. BT-Drucksache 15/5975, S. 41, S. 396. 
Staatsminister Volmer war beteiligt. Er verantwortet ihn mit. (...) Er hat ihn (jedoch) nicht geschrieben und er hat ihn erst recht nicht verfügt und erst recht nicht eigenmächtig. “42 Auch Fischer übernahm drei Tage später bei seiner Anhörung die politische Verantwortung für die Fehler seiner Mitarbeiter: „Wo Menschen sind, werden Fehler gemacht. Wenn in diesem Amt Fehler gemacht wurden, trage ich die Verantwortung für diese Fehler. Auch das gehört zur Ministerverantwortung. (...) Die Verantwortung liegt bei mir. Schreiben Sie hier rein: Fischer ist schuld. “43

Diese Aussage stellt den Höhepunkt auf der idealtypischen Skandalkurve nach Kolb dar, denn Volmer und Fischer reagierten mit ihrem öffentlichen Eingeständnis auf die Skandalisierung und trugen dazu bei, dass die „Kernerzählung“ des Skandals abgeschlossen und der Übergang in die Abschwungphase eingeleitet wurde. ${ }^{44}$ Dies lässt sich auch anhand des Aufkommens der erschienenen Artikel nachweisen. Mit Ausnahme eines kurzen Zwischenhochs im Juni 2005, als die Regierungsfraktionen den Ausschuss beendeten und die Opposition dagegen erfolgreich vor dem Bundesverfassungsgericht klagte, nahm die Berichterstattung nach Fischers Aussage kontinuierlich ab. Selbst als im Juli 2005 der damalige Innenminister Otto Schily (SPD) vor dem Ausschuss aussagte, fanden sich nur noch 35 Beiträge. Schily rückte erst nach der Aussage Fischers in den Mittelpunkt der öffentlichen Debatte. Im Gegensatz zu seinem Kabinettskollegen wies er im Ausschuss jegliche Verantwortung mit der Begründung von sich, er sei nicht der „oberste Sachbearbeiter im Ministerium“ 45 . Der Vorwurf, das Innenressort hätte mit eigenen Verfehlungen ebenso zur Visa-Affäre beigetragen, kann als geradezu idealtypischer Vorgang erklärt werden, denn nach der faktischen Überschreitung des Skandalhöhepunktes im April versuchte die Opposition, ein „angrenzendes Thema“ zu skandalisieren. Da das mediale Interesse an der Affäre bereits deutlich nachgelassen hatte, verlief dieser Versuch jedoch weitgehend im Sande. Im Wissen um diesen Umstand musste Schily auch keine öffentliche Reue mehr zeigen oder politische Verantwortung übernehmen.

Die Berichterstattung über den Auftritt Schilys vor dem Untersuchungsausschuss stellte zugleich den Übergang in die Marginalisierungs- beziehungsweise Rehabilitationsphase dar. Das Interesse der Medien sank rapide und bewegte sich in der zweiten Sommerhälfte 2005 auf einem vernachlässigbaren Niveau. Lediglich zur letzten Ausschusssitzung am 30. August gab es noch einmal eine leichte Zunahme in der Berichterstattung. Dabei zeigt sich - wie auch bei allen anderen, hier analysierten Untersuchungsausschüssen -, dass das Interesse der Medien an den eigentlichen Untersuchungsergebnissen der Abschlussberichte offenkundig gering ist. Dies spricht für die These, dass Untersuchungsausschüsse zu einem Gutteil von Inszenierung und politischer Skandalisierung leben.

\subsection{Atypische Skandalverläufe}

Zu den Untersuchungsausschüssen mit einem atypischen Skandalverlauf zählt der „HypoReal-Estate-Ausschuss" (vgl. Abbildung 6). Dieser wurde am 25. März 2009 von den dama-

42 Ebenda, S. 281.

43 Ebenda, S. $281 \mathrm{f}$.

44 Vgl. Steffen Burkhard, a.a.O. (Fn. 5), S. 199.

45 BT-Drucksache 15/5975, S. 281. 


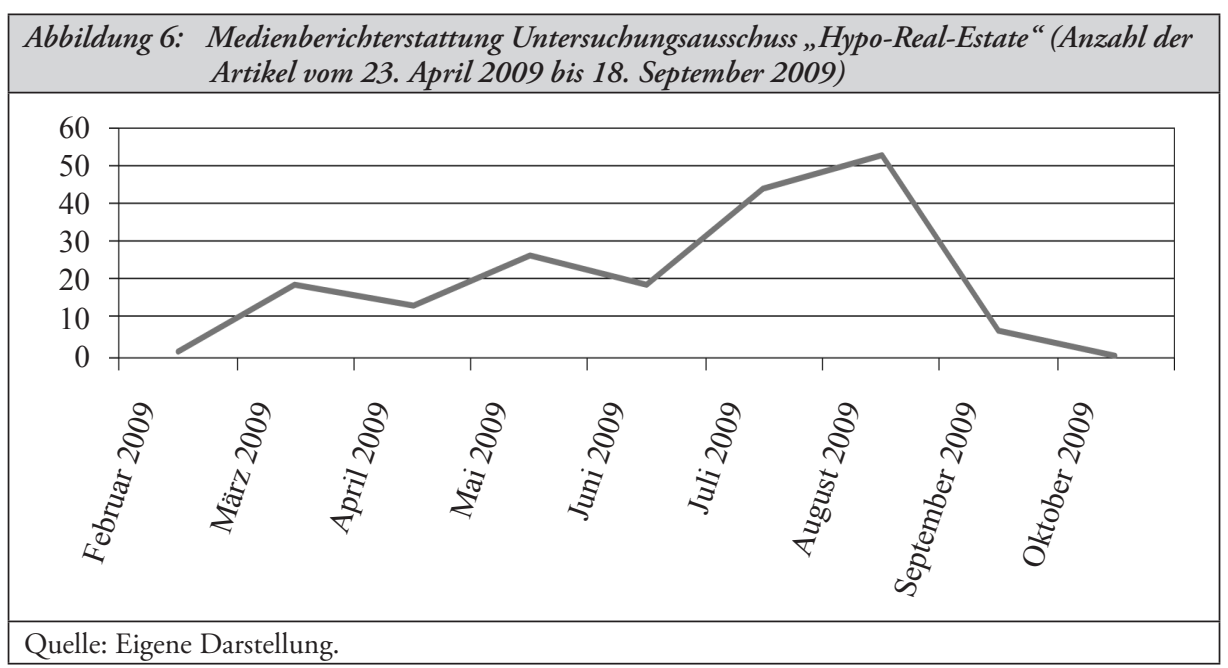

ligen Oppositionsfraktionen FDP, Die Linke und Bündnis 90/Die Grünen beantragt und sollte klären, „ob im Geschäftsbereich des Bundesministeriums der Finanzen (...) und der Bundesbank Erlasse, Weisungen, Fehleinschätzungen, öffentliche Äußerungen, Unterlassungen oder sonstige Handlungen zu den Missständen bei der Hypo Real Estate (...) beigetragen (...) haben“46. Die mediale Begleitung dieses Ausschusses nahm über einen langen Zeitraum stetig zu. Der Skandal nahm dabei zunächst - ähnlich wie im Visa-Untersuchungsausschuss - einen idealtypischen Verlauf. So erschienen im April 13, im Mai 26 und im Juni 19 Beiträge in den überregionalen Printmedien, in denen vor allem die mögliche Vorladung prominenter Zeugen diskutiert wurde. Dazu zählten der damalige Bundesfinanzminister Peer Steinbrück (SPD), dessen Staatssekretär Jörg Asmussen (SPD) sowie der damalige Vorstandsvorsitzende der Deutschen Bank, Josef Ackermann. Ihren Höhepunkt erreichte die Berichterstattung erst im August 2009 und damit einen Monat vor Abschluss der Untersuchungen. Diese relativ lange Aufschwungphase ist in erster Linie dadurch zu erklären, dass bis dahin keine Auftritte von prominenten Zeugen vor dem Ausschuss vorgesehen waren und es zu keiner personalisierten Skandalisierung einzelner Zeugen über die Medien kam. Weitere Gründe für die mediale Zurückhaltung dürften auch in der Komplexität der Materie sowie in der allgemeinen öffentlichen Empörung zu suchen sein, die sich damals in diffuser und pauschaler Weise vor allem gegen die Finanzmärkte und Banken richtete. Nicht umsonst änderte sich dies erstmalig Ende Juli 2009 mit der Vorladung von Josef Ackermann. Die Berichterstattung über seinen Auftritt erreichte dabei 44 Artikel in den überregionalen Printmedien. Diese Zahl stieg im August nochmals und erreichte mit 52 Beiträgen den Höhepunkt, als Asmussen und Steinbrück am 19. und 20. August vor dem Ausschuss erschienen. Auch in den Medien wurden beide Auftritte als „Höhepunkt“ beziehungsweise „entscheidende Sitzung “ des Ausschusses kommuniziert. ${ }^{47}$

46 BT-Drucksache 16/12480, S. 2.

47 Vgl. Sven Afhüppe, HRE-Ausschuss steht vor Höhepunkt, in: Handelsblatt vom 17. August 2009, S. 3; Jens Tartler, Promi-Auflauf im HRE-Ausschuss, in: Financial Times Deutschland vom 18. August 2009, S. 11. 

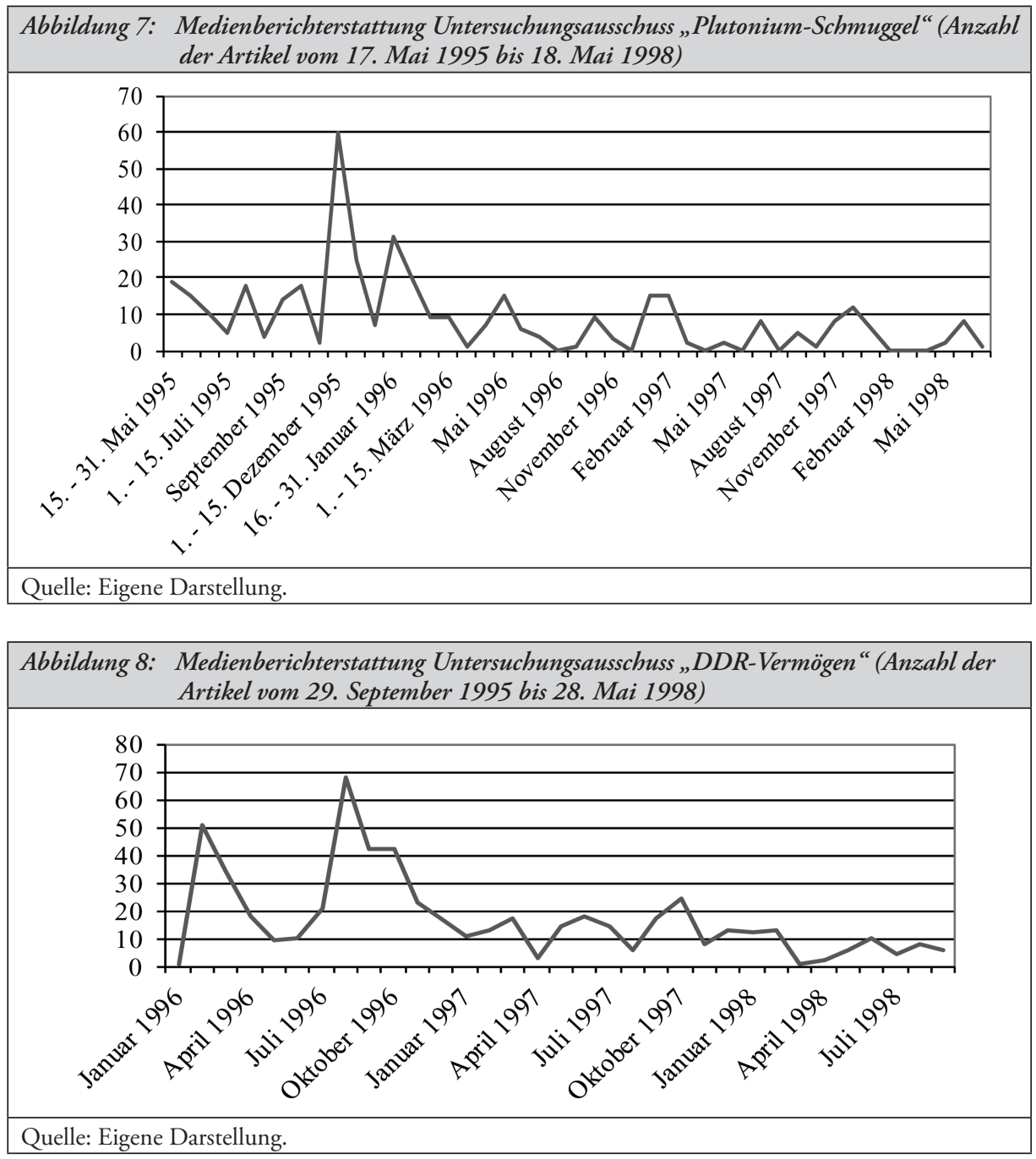

Betrachtet man den Verlauf der Medienrezeption als Ganzes, lässt sich feststellen, dass Abschwung- und Rehabilitationsphase nach Überschreitung der Klimax faktisch ausblieben. Die Anzahl der in den überregionalen Printmedien erschienenen Artikel sank im September 2009 auf sechs. Ein Grund dafür dürfte die damals kurz bevorstehende Beendigung der Ausschussarbeit gewesen sein, da der Untersuchungszeitraum aufgrund des Diskontinuitätsprinzips mit der Bundestagswahl am 27. September automatisch auslief. Auch dies zeigt einmal mehr, dass das Interesse der Medien an der publizistischen Aufbereitung und Bewertung entsprechender Abschlussergebnisse begrenzt ist. Da es zudem über die eigentliche Ausschussarbeit hinaus zu keiner weiteren nennenswerten Skandalisierung kam, setzt die mediale Skandalisierung offenbar das parlamentarische Verfahren voraus.

Weitere Gründe für einen atypischen Skandalverlauf lassen sich am Beispiel des Untersuchungsausschusses „Plutonium-Schmuggel“ aufzeigen (vgl. Abbildung 7), bei dem die 
mediale Berichterstattung - ähnlich wie beim Untersuchungsausschuss „DDR-Vermögen“ (vgl. Abbildung 8) - erst zu einem relativ späten Zeitpunkt ihren Höhepunkt erreichte. Dieser Skandal begann im August 1994 mit der Beschlagnahmung einer großen Menge Plutonium in einem aus Moskau kommenden Flugzeug am Münchener Flughafen. Nachdem in den Medien eine politische Dimension dieses Vorfalls zunächst nicht in Betracht gezogen worden war, berichtete „Der Spiegel“ im April 1995, dass diese „Schmuggel-Aktion“ von Bundesnachrichtendienst (BND) und bayerischen Ermittlungsbehörden inszeniert worden sei, um damit Einfluss auf die damals bevorstehende Landtagswahl in Bayern zu nehmen. Die Bundestagsfraktionen von Bündnis 90/Die Grünen und SPD beantragten daraufhin am 24. beziehungsweise 25. April 1995 die Einsetzung eines Untersuchungsausschusses, mit dem eine mögliche Verwicklung von Bundesbehörden in diesen Fall aufgeklärt werden sollte. Auch die Mehrheitsfraktionen von CDU/CSU und FDP stellten einen Antrag auf Einsetzung eines Untersuchungsausschusses - verfolgten damit jedoch eine gänzlich andere Intention: So vertrat der damalige CDU-Bundestagsabgeordnete Erwin Marschewski die Ansicht, dass „es sich nicht um einen Waffenskandal des Bundesnachrichtendienstes, sondern um einen Berichtsskandal des Spiegels “ 48 handle.

Dass der Skandalverlauf in der medialen Berichterstattung über diesen Ausschuss keine typische Aufschwungphase aufwies, erklärt sich aus zwei Gründen. Zum einen gelang es den Regierungsfraktionen, die Auftritte der prominenten Zeugen deutlich hinauszuzögern, so dass in den ersten sieben Monaten der Ausschussarbeit hauptsächlich Beamte und Sachverständige angehört wurden. Zum anderen ließ die Medienresonanz im August 1995 deutlich nach, um erst Ende September wieder anzusteigen, nachdem der Ausschuss nach der Sommerpause seine Arbeit wieder aufnahm. Dies belegt die These, dass es ohne den Ausschuss wohl nicht zu einer vergleichbaren Skandalisierung gekommen wäre.

Im Dezember 1995 erreichte der Skandal seinen medialen Höhepunkt, der gleich in zweifacher Hinsicht ungewöhnlich war: Neben dem Fehlen einer Aufschwungphase war auch der vernommene Zeuge nicht prominent, denn am 7. Dezember sagte der frühere V-Mann des BND Rafael „Rafa “Ferreras vor dem Ausschuss aus. In seiner Darstellung belastete er den damaligen Geheimdienstkoordinator im Bundeskanzleramt, Bernd Schmidbauer (CDU), sowie den BND mit der Aussage, den Schmuggel inszeniert zu haben. Zudem sprach er von einer Bedrohung durch die deutschen Behörden und einer Anstiftung zur Falschaussage. Die SPD erwog daraufhin, strafrechtlich gegen Schmidbauer vorzugehen. So bezeichnete der damalige innenpolitische Sprecher des SPD-Bundesvorstands, Ulrich Maurer, staatsanwaltschaftliche Ermittlungen als „unumgänglich“ “99. Der damalige BND-Chef, Konrad Porzner, erstattete seinerseits Anzeige wegen Falschaussage gegen Ferreras. All dies führte zu hoher Resonanz in den Medien. Als im Januar 1996 Schmidbauer und Porzner vor dem Ausschuss erscheinen mussten, war die Berichterstattung bereits deutlich zurückgegangen. Grund dafür war, dass Schmidbauer unmittelbar nach Ferreras' Aussage eine Pressekonferenz gegeben hatte, um zu dessen Vorwürfen Stellung zu nehmen. Aufgrund dieser persönlichen Initiative des Skandalisierten war der Skandalhöhepunkt somit zum Zeitpunkt seiner offiziellen Vernehmung im Ausschuss bereits überschritten. Bis zur Vorlage des Abschlussberichts im Juni 1998 ebbte die Berichterstattung über den Ausschuss stark ab. Auch vereinzelte Versuche einer medialen Personalisierung sollten nicht mehr aufgehen, wie der Auftritt des damaligen Bun-

48 BT-Plenarprotokoll 13/33 vom 26. April 1995, S. 2565.

49 „SPD nimmt sich Schmidbauer vor“, in: FR vom 15. Dezember 1995. 
deskanzlers Helmut Kohl vor dem Ausschuss am 13. November 1997 zeigte, der erstmals bereits im Januar 1996 von der Opposition beantragt worden war. ${ }^{50}$ Lediglich acht überregionalen Printmedien war dieses Ereignis noch ein eigener Beitrag wert.

\subsection{Ausbleibende Skandalisierung}

Zu den Fällen, die nur eine geringe mediale Resonanz aufweisen, zählen der Untersuchungsausschuss „HIV-Infektionen durch Blut und Blutprodukte“ (vgl. Abbildung 9) sowie der „Gorleben“-Untersuchungsausschuss (vgl. Abbildung 10). So erschienen beispielsweise zum HIV-Untersuchungsausschuss zwischen Oktober 1993 und Januar 1995 insgesamt lediglich 38 Beiträge in den überregionalen Printmedien. Hauptgrund für die geringe mediale Resonanz war vor allem die Thematik, die bereits eine interfraktionelle Einsetzung ermöglicht hatte. Nachdem bekannt geworden war, dass es bei Bluterkranken zur Übertragung des HIV-Virus durch Blutprodukte gekommen war, sollte der Ausschuss die Rolle und Verantwortung der „an der Herstellung, Verteilung und Überwachung Beteiligten“ untersuchen und prüfen, „welche Maßnahmen zur (...) Verbesserung der Sicherheit von Blut und Blutprodukten (...) geboten sind" 51 . Zudem sollten eventuelle Zahlungen an die Geschädigten geprüft werden. Das parteiübergreifende Interesse an der Aufklärung sorgte dafür, dass auf eine Skandalisierung weitgehend verzichtet wurde - auch als es zur Vernehmung prominenter Zeugen wie dem damaligen Gesundheitsminister Horst Seehofer (CSU) sowie Rita Süssmuth (CDU) und Heiner Geißler (CDU) kam, die dieses Amt in den 1980er Jahren bekleidet hatten. Auch der Abschlussbericht wurde fraktionsübergreifend beschlossen.

Im Gegensatz dazu weist die mediale Berichterstattung zum Gorleben-Untersuchungsausschuss - zumindest auf den ersten Blick - typische Merkmale einer erfolgreichen Skandalisierung auf. Allerdings bewegte sich das Ausmaß der Berichterstattung insgesamt auf einem sehr niedrigen Niveau. So erreichte sie im April 2010 ihren Höhepunkt mit nur

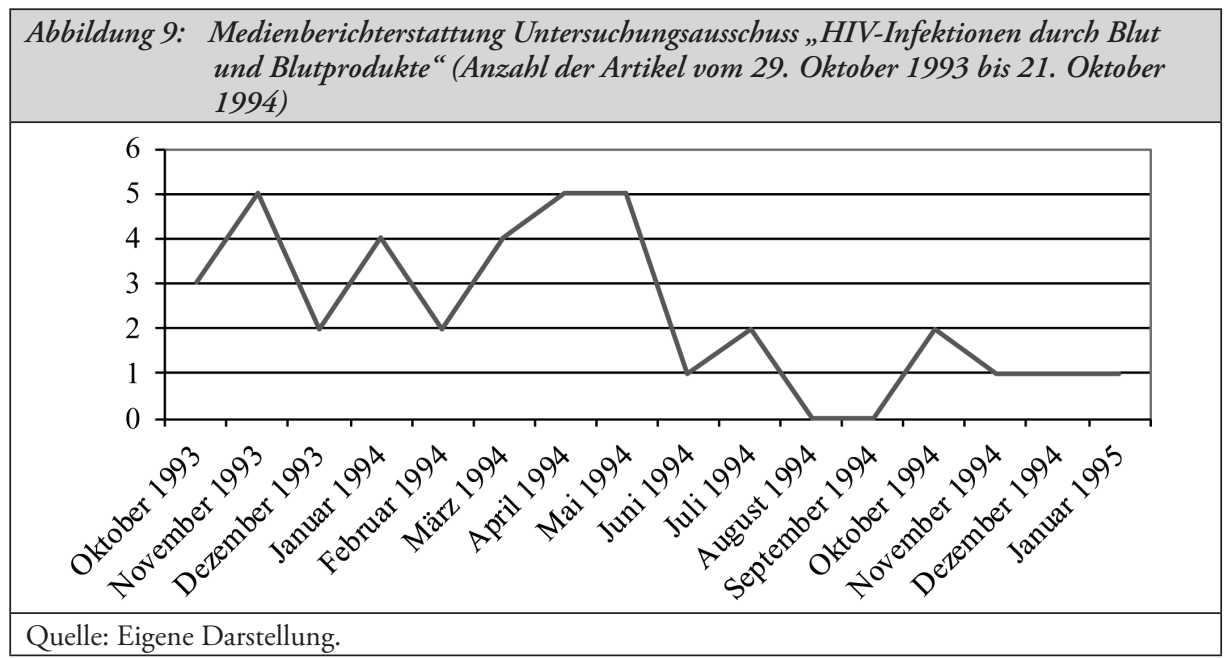

50 Vgl. BT-Drucksache 13/10800, S. 38.

51 BT-Drucksache 12/6048, S. 1. 


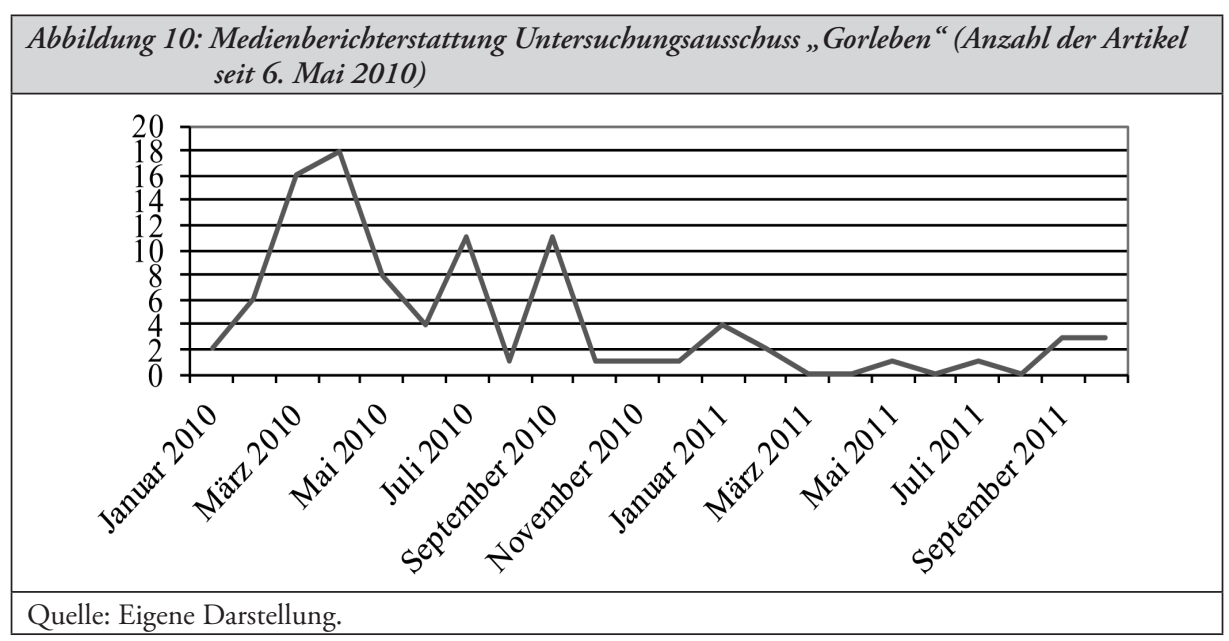

18 Beiträgen in den überregionalen Printmedien. Der Ausschuss war am 2. März 2010 von den drei Oppositionsfraktionen SPD, Bündnis 90/Die Grünen und Die Linke beantragt worden und sollte klären, weshalb sich die damalige Bundesregierung aus CDU/CSU und FDP in einem Kabinettsbeschluss von 1983 „bei der Suche nach einem Endlager für radioaktive Abfälle (...) ausschließlich auf die untertägige Erkundung des Standorts Gorleben “52 beschränkt hatte. Dahinter stand offenkundig ein tagespolitisches Kalkül, da in den Mehrheitsfraktionen zu diesem Zeitpunkt über eine Laufzeitverlängerung der Kernkraftwerke in Deutschland - auch als Wahlkampfziel - diskutiert wurde. So bekannte der SPD-Abgeordnete Sebastian Edathy im April 2010: „Wir verstehen diesen Ausschuss nicht als umweltpolitisches Fachforum. Wir werden die Vorkommnisse bei der Auswahl des Standortes mit der Grundsatzfrage verbinden, welche Zukunft die Kernenergie in Deutschland haben kann." 53 Die Skandalisierung zielte damit eigentlich im Kern auf die Fortführung eines zentralen Reformprojekts der rot-grünen Vorgängerregierung.

Dass die Skandalisierung jedoch ausblieb, lässt sich in dreifacher Weise begründen. Zunächst gelang es dem Ausschuss nicht, prominente Zeugen vorzuladen. Nach der Konstituierung wurden zwar diverse Namen diskutiert, die maßgeblichen Entscheidungsträger des Beschlusses waren damals jedoch bereits in einem fortgeschrittenen Alter und genossen zum Teil schon lange keine mediale Aufmerksamkeit mehr. Aktive Politiker wie die früheren Bundesumweltminister Sigmar Gabriel (SPD), Jürgen Trittin (Bündnis 90/Die Grünen) und Angela Merkel (CDU) wurden zwar immer wieder als mögliche Zeugen genannt, allerdings nicht vorgeladen. Unausgesprochen dürfte hier ein wechselseitiges parteitaktisches Einvernehmen nach dem do-ut-des-Prinzip eine Rolle gespielt haben, denn alle drei Minister hatten sich während ihrer Amtszeit nicht für einen anderen Standort stark gemacht. Ein weiterer Grund für die ausbleibende Skandalisierung dürfte auch in der Historie der Untersuchungsmaterie selber begründet liegen: Über dreißig Jahre zurückliegende Entscheidungen eignen sich offenbar nicht für eine mediale Skandalisierung - auch wenn Jahr für Jahr die zum Teil gewalt-

52 BT-Drucksache 17/888, S. 2.

53 Klaus Strattmann, Opposition macht Gorleben-Ausschuss zum Atomkraft-Tribunal, in: Handelsblatt vom 14. April 2010, S. 18. 
samen Bürgerproteste im Wendland für entsprechende Schlagzeilen sorgen. Das parteipolitische Konfliktpotenzial verlor der Ausschuss vollends mit dem von der schwarz-gelben Bundesregierung eingeleiteten „Wiedereinstieg in den Ausstieg der Kernenergie“ als Reaktion auf die nukleare Katastrophe in Japan im Frühjahr 2011. Für die Oppositionsfraktionen gab es damit faktisch kein Skandalisierungspotenzial mehr. Dementsprechend äußerte im September 2009 der CDU-Abgeordnete Reinhard Grindel, dass die Opposition „den Ausschuss nicht mehr als Vehikel (brauche), um über Atom und Endlager zu diskutieren“54.

\section{4. Öffentlichkeit als Bedingung der Skandalisierung}

Einen Sonderfall stellt der Ausschuss zur „Untersuchung zur Informationspolitik der Bundesregierung in Bezug auf Abläufe und Folgen des Bombardements von zwei entführten Tanklastern im Kundus-Fluss am 4. September 2009“, kurz: Kundus-Untersuchungsausschuss, dar (vgl. Abbildung 11). Da sich in diesem Fall der Verteidigungsausschuss gemäß Art. 45 a, Satz 2 GG als Untersuchungsausschuss konstituierte, kam es zu einem Konflikt zwischen den Mehrheitsfraktionen und der Opposition bezüglich öffentlicher Zeugenvernehmungen. So beschloss der Ausschuss zunächst im Dezember 2009, dass „Mitglieder der politischen Leitungsebene (Mitglieder der Bundesregierung, beamtete und parlamentarische Staatssekretäre, Abteilungsleiter und Pressesprecher) und militärischen Führung (Generalinspekteur und Stellvertreter) (...) grundsätzlich in öffentlicher Sitzung einvernommen “55 werden. Auf Antrag der Mehrheitsfraktionen wurde dieser Passus allerdings im Juni 2010 aus dem Einsetzungsbeschluss gestrichen, da $\$ 69$ Abs. 1 GO-BT für jeden Zeugen eine Einzelfallprüfung vorsehe. ${ }^{56}$ Die Medienberichterstattung zu diesem Untersuchungsausschuss ist deshalb von besonderem Interesse, da er zunächst prominente Zeugen in öffentlicher Sitzung anhörte, später jedoch die Öffentlichkeit - mit zwei Ausnahmen - ausschloss.

Die Berichterstattung nahm zunächst den typischen Verlauf mit Schlüsselereignis, Aufschwung-, Etablierungs- und Abschwungphase und erreicht ihren Höhepunkt im März 2010. Damals erschienen 83 Artikel zu den öffentlichen Vernehmungen des ehemaligen Generalinspekteurs der Bundeswehr, Wolfgang Schneiderhan, des vormaligen Staatssekretärs Peter Wichert sowie des ehemaligen Verteidigungsministers Franz Josef Jung (CDU). Als der damals amtierende Verteidigungsminister Karl-Theodor zu Guttenberg (CSU) einen Monat später zu seiner Aussage vor dem Ausschuss erschien, befand sich der Skandal zwar schon in der Abschwungphase, aufgrund der medialen Präsenz des Ministers erschienen trotzdem 36 Artikel in den überregionalen Printmedien. Nach dem Beschluss, den Ausschuss geheim tagen zu lassen, nahm die Berichterstattung jedoch signifikant ab. Ein abflauendes mediales Interesse kann dafür ganz offenkundig nicht verantwortlich gemacht werden, da die Opposition zuvor eine Gegenüberstellung von zu Guttenberg, Wichert und Schneiderhan gefordert hatte, was breit in den Medien diskutiert worden war. Dies belegt die These, dass eine Skan-

54 Felix Werdermann, Opas anhören oder Merkel bloßstellen, in: Der Freitag vom 15. September 2011, S. 4.

55 BT-Drucksache 17/7400, S. 14.

56 Vgl. dazu $\$ 69$ Abs. 1 GO-BT: „Die Beratungen der Ausschüsse sind grundsätzlich nicht öffentlich. Der Ausschuss kann beschließen, für einen bestimmten Verhandlungsgegenstand oder Teile desselben die Öffentlichkeit zuzulassen. Die Öffentlichkeit einer Sitzung ist hergestellt, wenn der Presse und sonstigen Zuhörern im Rahmen der Raumverhältnisse der Zutritt gestattet wird." 


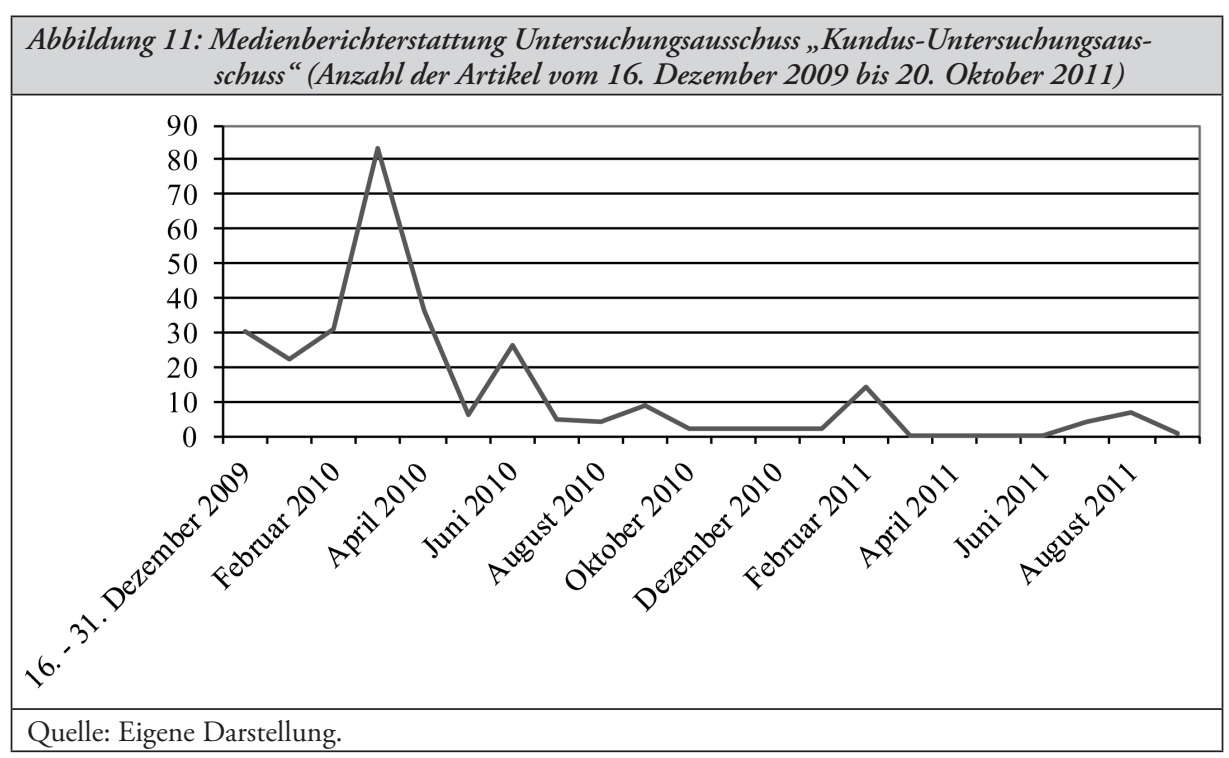

dalisierung nur über die Öffentlichkeit erfolgen kann. Tagen Untersuchungsausschüsse nicht öffentlich, verlieren sie an Attraktivität und Publikumswirksamkeit, die zur Erzeugung medialer Resonanz notwendig ist. Diese Annahme wird durch das kurzfristige Ansteigen des Medieninteresses im Februar 2011 unterstrichen, als Bundeskanzlerin Angela Merkel und SPD-Fraktionsvorsitzender Frank-Walter Steinmeier in öffentlicher Sitzung aussagten. Der dennoch vergleichsweise geringe Ausschlag lässt sich dabei mit der Kurzlebigkeit medialer Themenkonjunkturen begründen.

\subsection{Prominenz der Zeugen als Bedingung der Skandalisierung}

Am Beispiel der medialen Begleitung des BND-Untersuchungsausschusses lässt sich anschaulich eine Skandalisierung durch Personalisierung und Inszenierung - als Ausdruck von „Politainment" - aufzeigen (vgl. Abbildung 12): Nach dem Abschwung einer ersten Skandalkurve nahm die Berichterstattung ab September 2006 erneut kontinuierlich zu und erreichte im März 2007 einen zweiten Höhepunkt. Auch im weiteren Verlauf kam es immer wieder zu hohen Ausschlägen. Grund dafür war eine im Oktober 2006 vorgenommene Ausdehnung des Untersuchungsauftrages um den „Fall Murat Kurnaz“. Zudem verstanden es die Oppositionsfraktionen, das Interesse der Medien durch die geschickte Vorladung prominenter Zeugen stets erneut zu wecken.

Der BND-Untersuchungsausschuss war am 7. April 2006 auf Antrag der Oppositionsfraktionen FDP, Bündnis 90/Die Grünen und PDS/WASG eingesetzt worden. ${ }^{57}$ Er sollte

57 Parallel dazu konstituierte sich am 25. Oktober 2006 der Verteidigungsausschuss auf Antrag der Regierungsfraktionen CDU/CSU und SPD zu einem Untersuchungssauschuss. Der Untersuchungsauftrag galt der Rolle der Bundeswehr im Fall Murat Kurnaz und dem Einsatz von KSKKräften in Kandahar. Da er geheim zum BND-Untersuchungsausschuss tagte sowie einen ähnlichen Untersuchungsauftrag hatte, wurde er von den Medien kaum beachtet. 


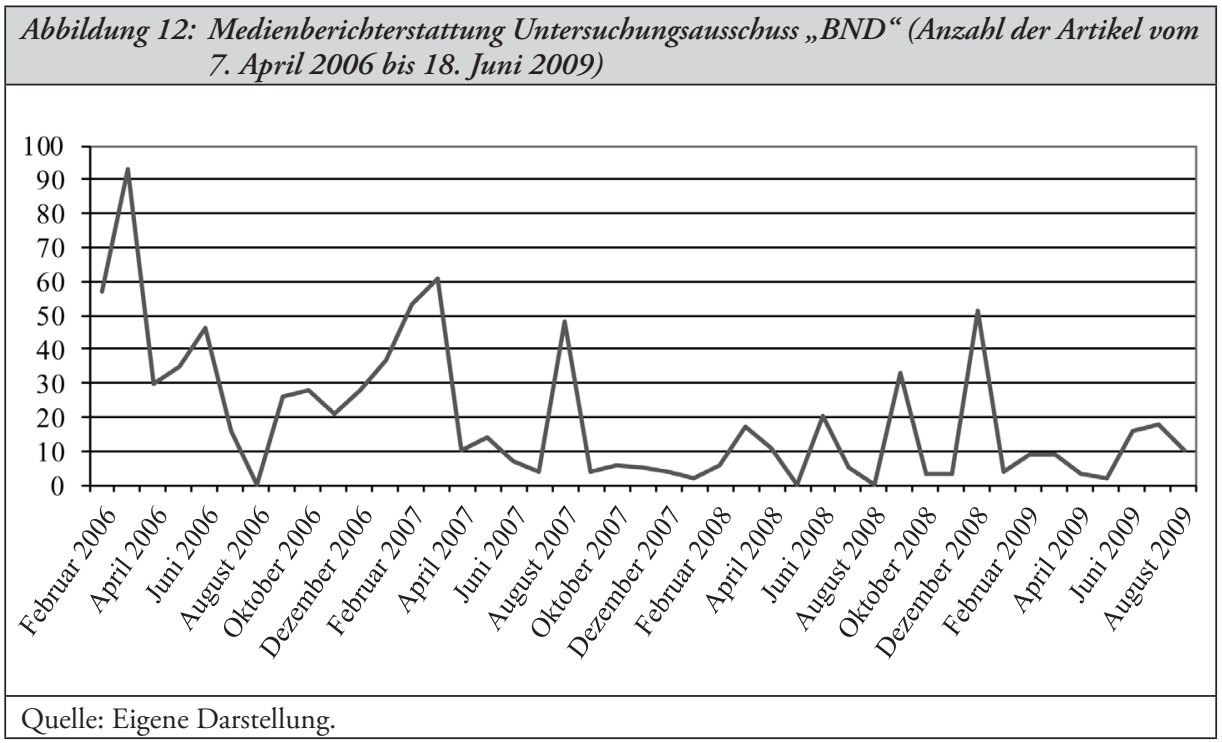

die Verstrickung der deutschen Geheimdienste in den Irakkrieg und in Entführungen von Terrorverdächtigen durch die CIA klären. Zudem sollte geprüft werden, welche Kenntnisse die Bundesregierung über geheime Gefangenentransporte der CIA über deutsches Hoheitsgebiet hatte. Da das Thema schon vor Arbeitsbeginn des Ausschusses in den Medien präsent war, erfolgte seine Einsetzung zu einem Zeitpunkt, als der Höhepunkt des medialen Skandals eigentlich schon überschritten war. Die Berichterstattung stieg jedoch nach Konstituierung des Ausschusses erneut an - ein Beleg für eine erneute erfolgreiche Skandalisierung über die Medien. Dies gründete zunächst wieder in einer Diskussion über die Vorladung prominenter Zeugen. Dabei ging es in erster Linie um den damaligen Außenminister und ehemaligen Chef des Bundeskanzleramtes Steinmeier, Ex-Bundeskanzler Schröder sowie die früheren Minister Schily und Fischer. Den ersten Höhepunkt erreichte die Berichterstattung im April 2006, als die öffentliche Anhörung zweier Geheimdienstmitarbeiter von der Bundesregierung verweigert wurde. Das mediale Interesse begann danach jedoch rasch zu sinken. Während der parlamentarischen Sommerpause im August 2006 erschien kein einziger Beitrag in den überregionalen Printmedien.

Der Eintritt in die zweite Skandalverlaufskurve erfolgte im September 2006. Dabei ging es um die Frage, ob der einen Monat zuvor nach Deutschland zurückgekehrte ehemalige Guantanamo-Häftling Murat Kurnaz schon im Jahr 2002 hätte freikommen können. Die Opposition beantragte deshalb im Oktober die Erweiterung des Untersuchungsmandats. So sollte geklärt werden, „welche Bemühungen im Fall Murat Kurnaz von der Bundesregierung unternommen wurden, um Murat Kurnaz Hilfe zu leisten und seine Freilassung zu erreichen, (...) ob und welche Angebote US-amerikanischer Stellen es für seine Freilassung gegeben hat, ob sie von deutscher Seite abgelehnt wurden oder ungenutzt blieben, wenn ja, aus welchen Gründen" 58 . Diese Ausweitung der Untersuchung führte in der Folge dazu, dass 
prominente Zeugen mehrfach vor dem Ausschuss erscheinen mussten. So kam es am 23. November 2006 zur ersten Zeugenaussage Otto Schilys zur Entführung eines deutschen Terrorverdächtigen durch die CIA im Jahr 2003. Mitte Dezember mussten Steinmeier und Fischer zu diesem Fall aussagen. Im Januar 2007 erschien Kurnaz vor dem Untersuchungsausschuss. Er schilderte detailliert Foltermethoden, die an ihm angewandt worden waren, und brachte damit Steinmeier in Bedrängnis. So berichtete die Süddeutsche Zeitung am 18. Januar, dass dieser noch 2005 eine Rückkehr Kurnaz nach Deutschland verhindert habe. ${ }^{59}$ Steinmeier wurde so zur maßgeblich skandalisierten Person und musste in der Folge noch vier weitere Male vor dem Ausschuss erscheinen. Allerdings versuchte auch er, den Ausschuss für seine Zwecke zu instrumentalisieren. So äußerte sein Sprecher Martin Jäger als Reaktion auf die Vorwürfe: „Wir hoffen, dass die Arbeit des Untersuchungsausschusses jetzt sehr zügig vorangeht und alle relevanten Zeugen gehört werden, damit Bundesminister Steinmeier dort rasch das Notwendige klarstellen kann."60 Der zweite Auftritt Steinmeiers wurde daraufhin vorgezogen.

Zum Höhepunkt der Etablierungsphase kam es im März 2007, als sowohl Steinmeier als auch Schily erneut vor dem Ausschuss erscheinen mussten. Ersterer erklärte, dass es „ein Angebot der amerikanischen Regierung zur Freilassung von Murat Kurnaz (...) - offiziell oder inoffiziell - nicht gegeben (habe), im Oktober 2002 nicht und später bis Anfang 2006 nicht"61. Die Medien beurteilten die Affäre daraufhin als „überstanden“, was sich auch in der Berichterstattung bemerkbar machte, die in der Folge deutlich nachließ. ${ }^{62}$ Die Kurve zeigt jedoch auch während der eigentlichen Rehabilitationsphase immer wieder Ausschläge. Mit zwei Ausnahmen sind diese auf weitere Vorladungen Steinmeiers zurückzuführen. Die Opposition verstand es mithin geschickt, eine vollständige Rehabilitation des damaligen Außenministers zu verhindern. Wie sehr die Arbeit von Parlamentarischen Untersuchungsausschüssen auch zu einer politischen Auseinandersetzung mit „Politainment“-Charakter werden kann, bei der die Inszenierung, Dramatisierung und Personalisierung die Aufklärungsarbeit überwiegen, wurde in dem Moment deutlich, als Steinmeier im Herbst 2008 zum SPD-Kanzlerkandidaten ernannt wurde. Hatte die Union ihn zuvor als Kabinettsmitglied der eigenen Koalition im Ausschuss und in den Medien verteidigt, änderte sich dies vor dessen fünfter und letzter Aussage im Dezember 2008. Der damalige Erste Parlamentarische Geschäftsführer der CDU/CSU-Fraktion, Norbert Röttgen, kündigte an, dass es „keine Schonung “ geben werde. ${ }^{63}$ Der damalige CSU-Landesgruppenchef Peter Ramsauer erklärte zudem, dass „Steinmeiers Verwicklung (...) zu einem Thema im kommenden Wahlkampf werden“ könne. „Je nachdem, was an Fakten zutage gefördert wird“, müssten entsprechende Schlussfolgerungen gezogen werden. Im schlimmsten Fall müsse „die SPD die Frage klären, ob Steinmeier Kanzlerkandidat bleiben kann"64.

59 Vgl. Hans Leyendecker, Rot-Grün blockierte Rückkehr von Kurnaz, in: SZ vom 19. Januar 2007, S. 1.

60 Angelika Hellemann, Stolpert Steinmeier über Bremer Taliban?, in: Bild am Sonntag vom 21. Januar 2007, S. 4a.

61 BT-Drucksache 16/13400, S. 181.

62 Vgl. Ansgar Graw / Joachim Peter, Im Zweifel für die Sicherheit, in: Die Welt vom 30. März 2007, S. 3.

63 CDU verlangt Aufklärung von Steinmeier, in: FAZ vom 17. Dezember 2008, S. 4.

64 Nikolai Fichtner / Friederike Tiesenhausen, Union knöpft sich Steinmeier vor, in: Financial Times Deutschland vom 18. Dezember 2008, S. 12. 


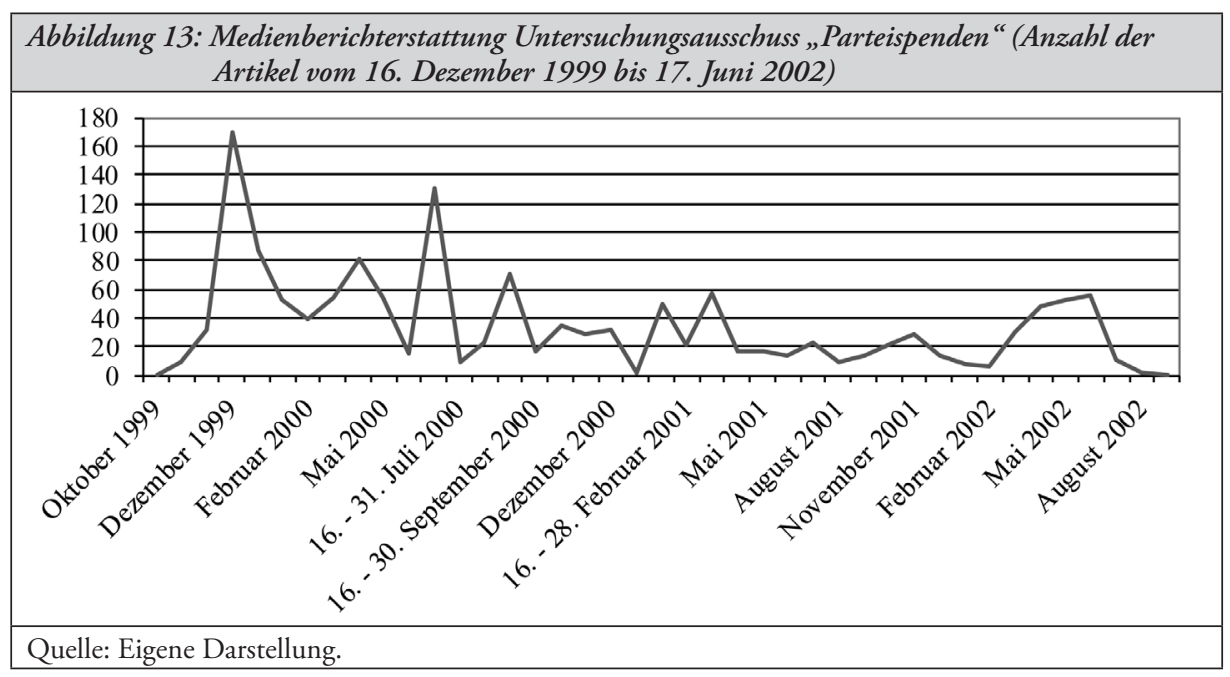

Ähnlich wie im BND-Untersuchungsausschuss zeigte sich auch in der Berichterstattung zum Untersuchungsausschuss „Parteispenden“, dass die Aussagen von Spitzenpolitikern immer wieder die höchste öffentliche Aufmerksamkeit erfahren. Zudem veranschaulicht der Ausschuss exemplarisch, wie Politiker Untersuchungsausschüsse - gerade im Kontext parteiinterner Auseinandersetzungen und persönlicher Konflikte - im Sinne einer Wahrheitskommission zur medialen Selbstverteidigung von Ruf und Ehre nutzen. Als Folge des CDU-Parteispendenskandals beantragten die damaligen Regierungsfraktionen von SPD und Bündnis 90/Die Grünen im November 1999 einen Untersuchungsausschuss, der klären sollte, ,inwieweit Spenden, Provisionen, andere finanzielle Zuwendungen oder Vorteile direkt oder indirekt an (...) Mitglieder und Amtsträger der ehemaligen von CDU/CSU und F.D.P. getragenen Bundesregierungen (...) geflossen sind beziehungsweise gewährt wurden, die dazu geeignet waren, politische Entscheidungsprozesse dieser Bundesregierungen (...) zu beeinflussen $(. . .)^{“ 65}$.

Im Mittelpunkt der Skandalisierung stand vor allem die Person des früheren Bundeskanzlers Kohl. Dieser versuchte, sich in der Anfangsphase durch verschiedene Interventionen als Herr des Verfahrens zu gerieren, in dem er selbst die Einberufung eines Untersuchungsausschusses verlangte. Er forderte den damaligen SPD-Fraktionsvorsitzenden Peter Struck in einer Plenardebatte am 24. November 1999 auf, ,dazu beizutragen, dass der von (der SPD) geforderte Untersuchungsausschuss unverzüglich eingesetzt wird, seine Arbeit noch vor Weihnachten beginnt und (ihm) die Chance gibt, dort noch vor Weihnachten auszusagen"66. Auch der Obmann der CDU/CSU-Fraktion im Ausschuss, Andreas Schmidt, verlangte im Januar 2000, „die Vernehmung Kohls unverzüglich auf die Tagesordnung zu setzen“67. Ein entsprechender Antrag wurde zunächst von der Ausschussmehrheit abgelehnt; dennoch wurde Kohl in der Folge vier Mal öffentlich verhört.

65 BT-Drucksache 14/2139, S. 1.

66 BT-Plenarprotokoll 14/72 vom 14. November 1999, S. 6516.

67 Helmut Lölhöffel, SPD benennt Zeugen für Ausschuss zur Spendenaffäre, in: FR vom 15. Januar 2000, S. 4. 
Mit seiner offensiven Vorgehensweise und den Versuchen einer Selbstmediatisierung konnte Kohl fortgesetzte Angriffe der Medien und des politischen Gegners nicht verhindern. Dies zeigte die mediale Resonanz zu seinen öffentlichen Anhörungen im Juni und Oktober 2000 sowie im Januar 2001 (vgl. Abbildung 13). Hauptgrund dafür war aber sein Verzicht auf ein öffentliches Schuldeingeständnis sowie die Übernahme von Verantwortung. So weigerte sich $K o h l$, Spendernamen zu nennen und verwies auf sein Ehrenwort. Im Ausschuss griff er die Regierungsfraktionen scharf an, die lediglich ein Interesse hätten, „ihn persönlich zu kriminalisieren und auf diese Weise seine Kanzlerschaft zu diskreditieren "68. Er sprach von einer „Welle von Verleumdungen und Diffamierungen, (...) Latrinengerüchten (...) und Hetzkampagnen "69. Zudem sah er seine politischen Verdienste nur unzureichend gewürdigt. Die Medien akzeptierten diese Argumentation jedoch nicht als öffentliche Läuterung. Die Berichterstattung blieb im Tenor negativ. ${ }^{70}$ Dies zeigt, dass eine Selbstmediatisierung dann nicht verfängt, wenn der Skandalisierte die moralischen Erwartungshaltungen von Öffentlichkeit und Medien nicht erfüllt. Dem politischen Gegner eröffnet sich damit die Möglichkeit, jederzeit neue Skandalisierungsversuche zu unternehmen.

\section{Fazit: Zwischen Skandalisierungsinstrument und institutionalisierter Warnfunktion}

Da, wie gezeigt, der Skandalisierungsgrad und -verlauf der einzelnen Ausschüsse divergiert, hängt eine „erfolgreiche“ Skandalisierung des politischen Gegners nicht - wie oft unterstellt - allein von der bloßen Einsetzung eines Untersuchungsausschusses, sondern auch von Intention und Vorgehensweise der involvierten Akteure, der Skandalisierten sowie den Medien ab. Bedeutung kommt außerdem einzelnen Schlüsselereignissen im Untersuchungsverlauf zu. Vor allem der Akt der Einsetzung eines Untersuchungsausschusses erzeugt mediale Resonanz. Aber auch eine über die Medien ausgetragene Diskussion über die mögliche Vorladung prominenter Zeugen sowie die eigentliche Anhörung dieser Personen erfahren erhöhte öffentliche Aufmerksamkeit. In allen Fällen besteht eine Korrelation zwischen dem Skandalhöhepunkt und der öffentlichen Anhörung prominenter Politiker. Ähnliche Ausschläge in der Berichterstattung lassen sich verschiedentlich in der Rehabilitationsphase nachweisen.

Voraussetzung für eine „erfolgreiche“ mediale Skandalisierung bildet zudem das Moment der Öffentlichkeit. Beim Kundus-Untersuchungsausschuss ließ beispielsweise das mediale Interesse bei geheimer Tagung des Ausschusses zwar nicht vollständig nach, verlor jedoch deutlich an Attraktivität - vor allem für die Opposition. An der ausbleibenden Skandalisierung, vor allem bei den Untersuchungsausschüssen „HIV-Infektionen durch Blut und Blutprodukte“ und „Gorleben“, wurde deutlich, dass auch der Untersuchungsgegenstand selber ein gewisses Maß an parteipolitischem Konfliktpotenzial und medialer Relevanz aufweisen

68 BT-Drucksache 14/9300, S. 362.

69 Volker Skierka, Der Untersuchungsausschuss, in: SZ vom 23. Februar 2001, S. 14.

70 Vgl. dazu Franziska Augstein, Wie man aus einem Elefanten eine Mücke macht. Der Untersuchungsausschuss und die CDU-Spendenaffäre, in: Merkur - Zeitschrift für europäisches Denken, 55. Jg. (2001), S. 382; Markus Deggerich, Spendenskandal: Alle verkohlt, in: Spiegel Online vom 13. Dezember 2001, http://www.spiegel.de/politik/deutschland/spendenskandal-alle-verkohlta-172605.html (Abruf am 8. März 2013). 
muss, damit es zu einer Skandalisierung kommen kann. Stimmen Mehrheits- und Oppositionsfraktionen in grundsätzlichen Fragen weitgehend überein, ist parteitaktisches Kalkül über die Bande der Medien kaum möglich.

In der Vergangenheit wurden Untersuchungsausschüsse auch von den skandalisierten Akteuren selber als Plattform genutzt. Auch wenn sich eine Skandalisierung dadurch nicht gänzlich verhindern lässt, sind doch Verlauf und Prozess - zum Beispiel durch das Timing und den inhaltlichen Gehalt von Aussagen - in einem gewissen Rahmen beeinflussbar. Dass die Forderung nach einer möglichst raschen Anhörung als verschiedentlich genutzte Selbstverteidigungsstrategie unterschiedlich erfolgreich war, zeigte sich bei Joschka Fischer im VisaUntersuchungsausschuss, Frank-Walter Steinmeier im BND-Untersuchungsausschuss und Helmut Kohl im „Parteispenden“-Ausschuss. Fischer überstand das Verfahren ohne erneute Skandalisierung in der Rehabilitationsphase, da er die vollständige politische Verantwortung für den festgestellten Missstand übernahm. Steinmeier verteidigte hingegen sein Handeln im Fall Murat Kurnaz. Dies wurde zwar von den Medien akzeptiert, allerdings kam es durch wiederholte Vorladungen zu weiteren Skandalisierungsversuchen durch die Opposition. Kohl nutzte den Ausschuss vor allem, um seine politischen Gegner anzugreifen. Dieses Verhalten wurde von den Medien nicht goutiert. So kam es nie zu einer echten medialen Rehabilitation: Kohl hat bis heute seine Reputation nicht vollständig wieder herstellen können.

In der Summe lässt sich feststellen, dass es in der parlamentarischen Praxis in elf von dreizehn untersuchten Fällen zu Formen einer Skandalisierung über die Medien kam. Immer wieder wurden Untersuchungsausschüsse - wie auch diverse Aussagen von Politikern belegen -, von der Opposition gezielt zur Skandalisierung der Mehrheitsfraktionen genutzt. Das Wechselspiel zwischen den Akteuren wird dabei erkennbar durch eine Medienlogik geprägt, die von Formen der Inszenierung und Personalisierung lebt. In einem eigentümlichen Widerspruch dazu steht das häufig anzutreffende Desinteresse der Medien an den eigentlichen Untersuchungsergebnissen, die dem Bundestag und der Öffentlichkeit zum Verfahrensabschluss präsentiert werden. Dies verweist auf die zum Teil extrem kurzen medialen Themenzyklen und die Spannungslage zwischen zeitaufwendigen und detailreichen parlamentarischen Einzelfalluntersuchungen einerseits sowie der kurzen Aufmerksamkeitsspanne der breiten Öffentlichkeit andererseits.

Wenn Parlamentarische Untersuchungsausschüsse aufgrund der ausgeprägten Minderheitsrechte und der erhöhten medialen Aufmerksamkeit immer wieder als Instrument zur Skandalisierung der Mehrheitsfraktionen genutzt werden, stellt sich die Frage, ob sie damit die Funktion eines parlamentarischen Kontrollinstruments hinreichend erfüllen. So ließe sich angesichts knapper werdender Haushaltsressourcen die Verhältnismäßigkeit von Kosten und Ertrag bezweifeln, da ein solcher Ausschuss immer administrative Kapazitäten bindet - sei es in der Bundestagsverwaltung oder bei den einzelnen Abgeordneten. Demgegenüber steht jedoch eine institutionalisierte Warnfunktion, die diese parlamentarische Kontrollinstitution - allein aufgrund ihrer verfassungsrechtlichen Verankerung - präventiv entfaltet. Die selbsterzieherischen und selbstreinigenden Effekte, die die Einsetzung eines Ausschusses mit sich bringen kann, werden nicht vollends durch die dominante Medienlogik verzerrt, sondern eröffnen einzelnen Abgeordneten durchaus auch Profilierungsmöglichkeiten. 